\title{
CRECIMIENTO ECONÓMICO: CONVERGENCIA Y DIVERGENCIA
}

\author{
ECONOMIC GROWTH: CONVERGENCE AND DIVERGENCE \\ CRESCIMENTO ECONÔMICO: CONVERGÊNCIA E DIVERGÊNCIA
}

\section{Daniel Villalobos Céspedes ${ }^{1}$}

\begin{abstract}
Resumen
Esta investigación examina las Leyes fundamentales del capitalismo según Piketty en "Capital in the Twenty-First Century" (2014) para develar el poder explicativo de la desigualdad entre la tasa media anual de retorno del capital y la tasa de crecimiento de la producción, como fuerza fundamental para la divergencia. Se cuestiona el supuesto crítico del autor tal que $r>g$ y se duda de sus conclusiones, para afirmar que la convergencia-divergencia es un fenómeno innato de los procesos de producción y distribución. El análisis sugiere que el núcleo de la inequidad podría no residir en la elasticidad-de-composición de los recursos, con influjo del avance tecnológico. Además, pretende esta investigación contribuir a una mejor comprensión de las fuerzas con influjo en el crecimiento económico y en la distribución del ingreso, lo cual podría brindar un importante aporte a la ciencia económica.
\end{abstract}

Palabras clave: Piketty; leyes del capitalismo; retorno; salario

\begin{abstract}
Piketty's fundamental laws of capitalism in his book "Capital in the Twenty-First Century" (2014) are analyzed to explore the explanatory power of the inequality between average annual rate of return on capital and the rate of growth of production, as a fundamental force for
\end{abstract}

Doi: https://doi.org/10.15359/eys.24-55.3

Fecha de recepción: 06-09-2018. Fechas de reenvíos: 10-09-2018, 18-12-2018, 13-01-2019, 05-12-2019. Aceptado el 12-03-2019. Publicado el 13-03-2019.

${ }^{1}$ Economista, politólogo, catedrático en Universidad de Costa Rica (UCR) y Universidad Nacional (UNA), Costa Rica. Correo electrónico: daniel.villalobos.cespedes@una.ac.cr ORCID https://orcid.org/0000-0002-9023-2096

Daniel Villalobos Céspedes

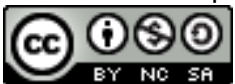

Revista Economía y Sociedad by Universidad Nacional is licensed under a CreativeCommons Reconocimiento-NoComercial- 
divergence. The author's $r>g$ crucial assumption and his conclusions are questioned. The convergence-divergence phenomenon is rather believed to be an innate phenomenon of the production and distribution processes. This analysis suggests that the core of inequality may not reside in the elasticity-of-resource-composition, influenced by technological advance. Furthermore, this research will try to contribute to a better understanding of the forces influenced by economic growth and income distribution, which could provide a major contribution to the economic sciences.

Keywords: Piketty; laws of capitalism; return; wage

\section{Resumo}

Esta pesquisa examina as Leis Fundamentais do capitalismo segundo Piketty em "Capital in the Twenty-First Century" (2014) para revelar o poder explicativo da desigualdade entre a taxa média anual de retorno do capital e a taxa de crescimento da produção, como força fundamental para a divergência. $O$ suposto crítico do autor é questionado de tal forma que $r>g$ e suas conclusões são duvidadas, para afirmar que a convergência-divergência é um fenômeno inato dos processos de produção e distribuição. A análise sugere que o núcleo da desigualdade poderia não residir na elasticidade da composição dos recursos, influenciada pelo progresso tecnológico. Além disso, esta pesquisa visa contribuir para uma melhor compreensão das forças que influenciam o crescimento econômico e a distribuição de renda, o que poderia fornecer uma contribuição importante para a ciência econômica.

Palavras-chave: Piketty; leis do capitalismo; retorno; salário

\section{Introducción}

Thomas Piketty, profesor de la Escuela de Economía de Paris, Francia, destelló en el año 2014 con su obra "Capital in the Twenty-First Century", "dedicada esencialmente a comprender la dinámica histórica de la riqueza y el ingreso" (Piketty, 2014, p. vii). ${ }^{2}$ Con base en datos de una

2 “El Fenómeno Piketty” (Goldhammerr, 2017) es una excelente reseña del autor y del libro en cuestión. También en "Economist Receives Rock Star Treatment" (Schuessler, 2014) esbozó el fervor académico, político y de mercado en torno a ese autor.

26

Daniel Villalobos Céspedes

(c) (1) 8 (9)

Revista Economía y Sociedad by Universidad Nacional is licensed under a CreativeCommons Reconocimiento-NoComercial- 
veintena de países, Piketty (2014) relata la evolución de la distribución de la riqueza - eje central de sus investigaciones - desde el siglo dieciocho (p. 1). El supuesto crítico del autor es que la tasa de retorno $(r)$, como porcentaje del retorno $(R)$ sobre el capital $(K)$ o valor total de la riqueza privada - incluida la acumulación por herencia -, excede la tasa de crecimiento del producto 0 del ingreso, o tasa de crecimiento de la economía $(g)$, debido a un conjunto de fuerzas de divergencia - no perpetuas - asociadas con procesos - de orden político y económico - de concentración y acumulación de la riqueza cuando el crecimiento es débil, sobre todo en mercados de capital prefectos. Ese supuesto cimienta la principal fuerza de divergencia en la propuesta teórica del autor: $r>g$ (pp. 23-27, 52), que emerge del análisis de las Leyes fundamentales del capitalismo (pp. 52-55, 166-168).

Entre sus críticas, Krugman (2014) describió la obra como "un libro verdaderamente magnífico...un trabajo increíble" que "cambiará tanto la forma en que pensamos la sociedad y en la que hacemos economía" y afirmó que "Piketty ha transformado nuestro discurso económico; nunca hablaremos acerca de la riqueza y la inequidad de la misma manera que solíamos hacerlo". Krugman atribuye la inequidad, en buena parte, a las acciones de los gobiernos (impuestos y transferencias) y al mercado, empero defiende los altos ingresos de los ejecutivos financieros y sugiere que el analisis de Piketty "carece de rigor analítico” en este ámbito. Krugman (2015) reiteró que el libro de Pikety es una "obra maestra... un libro poderoso, bellamente escrito", pero una versión ligeramente revisada de la publicada en el año 1997.

Otras autorías cuestionaron aspectos técnicos y metodológicos y la capacidad explicativa y predictiva de la propuesta de Piketty. Solow (2014) sugirió un ambiguo uso de las categorías riqueza y capital en Piketty (2014) , quien reveló usar "como términos intercambiables" y útil "para simplificar el texto", y computó así riqueza o capital nacional "como el valor total de mercado" (p. 47) de las posesiones negociables en un país. Solow (2014) recalcó la diferencia entre contribución del capital y del trabajo en el ingreso nacional, y afirmó que la persona trabajadora "se come su salario, no su contribución al ingreso nacional" (p. 2) y que vivir en una sociedad donde el salario real y la productividad se estancan, pero la participación del trabajo disminuye, podría generar ventajas sociales y políticas para que algunas personas acumulen una mayor parte del ingreso nacional; como consecuencia, "el-rico-se-hace-más-rico" (p. 3).

Raval (2017) cuestionó las Leyes del capitalismo y la elasticidad de substitución capital-trabajo, y se inclinó por atribuir a la globalización la caída en la participación del trabajo en el ingreso a la pérdida de empleos, disminución de salarios, o ambos, en los países importadores, en especial en las industrias intensivas en trabajo y al progreso tecnológico, causantes de elasticidad de substitución menores que la unidad. Naidu (2017) rechazó la valoración del capital a precios de mercado y fijar $(r>g)$, y asemeja Piketty con Marx, quien limitó el salario al nivel de subsistencia; supuestos que se asumen como un hecho histórico. Pikety (2017) otorga razón a Naidu: $(r>g)$ 
"siempre se cumple, y surge mecánicamente de supuestas leyes psicológicas universales" (p. 642).

Galbraith (2014), invocando a Marx, arremetió contra el uso del concepto de capital. Señala que la razón capital/ingreso depende fuertemente del flujo de valor en el mercado y no de su condición física. En su criterio, durante la I Guerra Mundial hubo poca destrucción física del capital respecto de la segunda Guerra Mundial, y durante la Gran Depresión hubo principalmente pérdida de valor de mercado del capital. Galbraith insiste en que ese evento se refleja en $(r>g)$, pero desconoce el origen de $(r)$ y destaca la insuficiencia explicativa del fenómeno desigualdad cuya "evolución...no es un proceso natural", y duda del aumento de impuestos como solución. Mankiw (2015) refutó las principales conclusiones de Piketty debido a que "varios enlaces en la cadena de argumentos de Piketty son especialmente frágiles" (p. 43), y analizó la validez de ( $r$ > $g$ ) con un modelo sencillo, y sugiere un impuesto progresivo al consumo, un buen sistema de educación y salud, promover el ahorro y permitir que las personas trabajadoras se conviertan en capitalistas (Mankiw, 2015). Stiglitz ( $\underline{2015}$ ) estilizó en distintos modelos fuerzas centrípetas y centrífugas micro y macro - demografía, explotación, recursos naturales, educación, poder de mercado - que podrían incitar mayor o menor desigualdad. Stiglitz (2015) sugirió que se requiere $[s r>g ;(0 \leq s<1)]$, donde $s$ es la tasa de ahorro en la economía, para que la riqueza de un capitalista siempre crezca; capital y riqueza no son análogos.

La propuesta de Piketty se acerca a las de Harrod (1939) y (1960) y Domar (1946) y (1953), se distancia de Cobb y Douglas (1928), Swan (1956), Solow (1956) y (1957) y Arrow, Chenery, Minhas y Solow (1961) y difiere de Marx (Villalobos, 2010). Solow (1956) sentenció:

Toda teoría depende de supuestos que no son del todo ciertos... Un supuesto 'crucial' es aquél en que las conclusiones dependen sensiblemente, y es importante que los supuestos cruciales sean razonablemente realísticos. Cuando los resultados de una teoría parecen fluir específicamente de un supuesto crucial especial, entonces si el supuesto es dudoso, los resultados son sospechosos. (p. 65$)^{3}$

Con base en ese principio, la presente investigación se propone develar las fortalezas explicativas de la divergencia en la convergencia. Para tal efecto, se adiciona la clásica preocupación de Cobb y Douglas (1928) sobre el problema producción-distribución:

\footnotetext{
3 "All theory depends on assumptions which are not quite true. That is what makes it theory...A "crucial" assumption is one on which the conclusions do depend sensitively, and it is important that crucial assumptions be reasonably realistic. When the results of a theory seem to flow specifically from a special crucial assumption, then if the assumption is dubious, the results are suspect" (Solow, 1956, p. 65).
}

28

Daniel Villalobos Céspedes

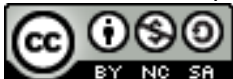

Revista Economía y Sociedad by Universidad Nacional is licensed under a CreativeCommons Reconocimiento-NoComercial- 
¿Podemos aclarar el problema de si los procesos de distribución son o no modelados por los procesos de producción de valor? (pp. 139-140)

Así como sus sugerencias:

Deberíamos (1) estar preparados para idear fórmulas que no necesariamente se basen en la "contribución" relativa de cada factor al producto total, sino que expliquen variaciones anuales, y (2) que eliminen hasta donde sea posible el elemento tiempo de los procesos. (p. 165) ${ }^{4}$

y la propuesta de Harrod (1939) y (Harrod, 1960) que motiva estudiar cómo operan las fuerzas con influjo en la tendencia del crecimiento y sus efectos en el cambio sostenido en la distribución del ingreso.

Esta investigación propone una teoría del crecimiento económico y distribución del producto enfocada en el fenómeno convergencia-divergencia. El principal aporte es valuar ese fenómeno según evolución de la productividad-ingreso de los recursos. El primer apartado analiza, esencialmente, el supuesto crucial en la obra de Piketty (2014) a partir de las formulaciones que sustentan sus leyes fundamentales del capitalismo. El segundo apartado plantea la función agregada de la distribución del ingreso, de donde emerge la ruta-de-distribución de la que se ocupa el siguiente apartado. A continuación, se desarrolla el modelo de la función compuesta del crecimiento económico, el cual se basa en nuevas formulaciones no registradas por la bibliografía en ese tema. En el quinto y el sexto apartado se completa el modelo con las funciones de costo y de valor de la producción y la función de ganancia. Al término de la investigación surgen las conclusiones y las referencias. Finalmente, se anexa un ejercicio hipotético que pretende evidenciar el poder explicativo del modelo propuesto.

\section{De los supuestos cruciales de Piketty}

\section{La primera ley del capitalismo}

Piketty (2014) calcula el retorno $(R)$ como un porcentaje $(r)$ del capital $(K)$ a cada instante: ${ }^{5}$

\footnotetext{
4 "May we secure light upon the question as to whether or not the processes of distribution are modeled at all closely upon those of the production of values? (Cobb \& Douglas, 1928, pp. 139-140)..."We should (1) be prepared to devise formulas which will not be necessarily be based upon constant relative "contribution" of each factor to the total product, but which will allow for variations from year to year, and (2) will eliminate so far as possible the time element from the process" (Cobb \& Douglas, 1928, p. 165).

${ }^{5}$ La antítesis de esta medición la planteó Marx (1984) con el concepto de tasa de plusvalor o de explotación del trabajo $\left(p v^{\prime}\right)$ como porcentaje de la ganancia $(G)$ respecto del total del capital variable o salarios $(s)$ pagado al trabajo $(T)$, tal que: $p v^{\prime}=\frac{G}{s T}$. Un análisis crítico al respecto se encuentra en (Villalobos, 2010).
} 
(1) $r=\frac{R}{\mathrm{~K}}$

y la participación media de $(K)$ en el ingreso anual $(Y)$ :

(2) $ß=\frac{K}{Y}$

Al insertar la ecuación (2) en (1) resulta $\left[R=r \beta Y \therefore r \beta=\frac{R}{Y}\right]$ y sea $\alpha=\frac{R}{Y}$ para definir la primer Ley de Piketty: 6

(3) $\alpha=r \beta$

La divergencia entre $(\alpha, \beta)$ denota la fuerza fundamental $(r>g)$ de Piketty, donde $(g)$ es la tasa de crecimiento de la economía. Si $K=ß Y$, al derivar resulta $(k K=ß g Y)$ y al substituir la ecuación (1) $\left(ß g Y=\frac{1}{r} k R\right) \therefore$

(4) $r=\frac{\alpha}{\beta} \frac{k}{g}$

Esa ecuación explica el enunciado de Piketty acerca de las formas-U de $(\alpha, \beta)$; si $ß$ disminuye dado $k>0$ podría deberse a un incremento en la productividad y, si $\left(\frac{\alpha}{\beta}>\frac{k}{g}\right),(r)$ crece cuando (ß) cae, y $(k=g)$ implica la ecuación (3). Así, $(r)$ podría tender a decrecer a largo plazo, y la defenza de Piketty (2017) frente a la crítica de Naidu (2017) no se sostiene.

\section{La segunda ley del capitalismo}

La primer ley contiene la segunda al relacionar la tasa de crecimiento del ahorro en la economía $(s) \operatorname{con}(g)$ :

(5) $ß=\frac{s}{g}$

Sea $(K=ß Y)$ y al substituir (4) resulta:

(6) $K=\frac{s}{g} Y$

${ }^{6}$ Más simple: si $\alpha=\frac{R}{Y}$ al insertar la ecuación (1) y dada la definición en (2), resulta (3).

30

Daniel Villalobos Céspedes

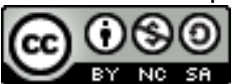

Revista Economía y Sociedad by Universidad Nacional is licensed under a CreativeCommons Reconocimiento-NoComercial- 
La acumulación de capital es influida por $\left(\frac{s}{g}\right)$ tal que $\frac{d K}{d Y}=\frac{s}{g}$ y $(d K, d Y)$ miden cambios brutos en $(K, Y)$ respectivamente $\mathrm{y},\left[K_{t}=K_{t-1}+d K\right]$ y $\left[Y_{t}=Y_{t-1}+d Y\right]$ para que $\left[\frac{K_{t}}{Y_{t}}=\frac{K_{t-1}}{Y_{t-1}}+\frac{d K}{d Y}\right] \therefore$ $\left[\frac{K_{t}}{Y_{t}}=\frac{K_{t-1}}{Y_{t-1}}+\frac{s}{g} \frac{K_{t-1}}{Y_{t-1}}\right] \therefore \frac{K_{t}}{Y_{t}}=\left[1+\frac{s}{g}\right] \frac{K_{t-1}}{Y_{t-1}} \mathrm{y}$ así:

(7) $\beta_{t}=\left[1+\frac{s}{g}\right] ß_{t-1}$

La segunda ley podría denotar evolución de (ß) según $(s, g)$ (Piketty, 2014).

Empero, $\left[\frac{d K}{d Y}=\frac{s}{g}\right] \therefore\left[\frac{k}{g} \frac{K}{Y}=\frac{s}{g} \therefore \frac{k}{g} ß=\frac{s}{g}\right]$ tal que:

(8) $ß=\frac{s}{k}$

y $\left(\frac{s}{k}\right)$ es la elasticidad-inversión del ahorro. Si $(s=k)$, resulta $\beta_{t}=\left[1+\frac{k}{g}\right] \beta_{t-1}, \mathrm{y}$ al equiparar con la ecuación (7) - o directamente al derivar $K=ß Y$ - resulta $\left[\frac{s}{g}=\frac{s}{k} \therefore k=g\right]$, que define el equilibrio ahorro-acumulación-ingreso en la economía. $(k)$ destaca la adición de capital incluida la reposición por depreciación y depende de $g$, la fuente de $s$. Podría suceder $(k \neq g)$, según condiciones micro y macroeconómicas; $(k)$ no necesariamente estimula $g$, pero $[s>(g, k)]$ indicaría (ß) creciente. Al insertar la ecuación (5) en (4) se obtiene:

(9) $r=\alpha \frac{k}{s}$

Si $(s>k)$ dado $(\alpha),(r)$, tiende a disminuir debido a la escaza inversión en $(K)$ y la caída en su productividad, pero si esta aumenta, $(r)$ crece. Por la ecuación (9) $\alpha k=s r$ y por las ecuaciones (3) у (5) $\alpha g=s r$ y $\alpha k=s r$ de donde $g=k$ y de (Stiglitz, 2015) $[s r>g=\alpha k>g]$ equivale a $\left[\alpha \frac{k}{s}>g\right]$ idéntico a $\left[r>g=\alpha \frac{k}{s}>g\right]$ en (Piketty, 2014). En ambos autores $\left(\frac{\alpha}{\beta}>g\right)$, por consiguiente, si $\left(\frac{\alpha}{\beta}=g\right)$ entonces $(\alpha=g ß)$ implica que $(R>g K)$ y, ceteris paribus, el retorno depende del crecimiento de la economía. Pero si $(R>g K)$, existen en la economía poderosos factores exógenos que actúan al margen de la producción global o, simplemente, el problema de confundir la riqueza con el capital (Solow, 2014). 


\section{Composición de los recursos y distribución del ingreso}

Piketty (2014) sugirió que a inicios del siglo veintiuno la participación de $(K, T)$ en $(Y)$ varió modestamente a favor del trabajo, respecto del siglo diecinueve, y se propuso analizar exclusivamente la evolución de la contribución del capital en el ingreso en vez de en su participación. Así, al evadir la elasticidad-de-composición de los recursos, el supuesto crucial de ese autor es frágil.

\section{La función de distribución}

Si $(Y)$ se distribuye en la forma de retorno $(R)$ para el capital $(K)$ y de salario $(S)$ para el trabajo $(T)$, la función de distribución es:

(10) $Y=F(R, S)$

tal que $1=F\left(\frac{R}{Y}, \frac{S}{Y}\right)$, y sea $\alpha=\frac{R}{Y}$ la participación del capital en el ingreso y $1=F\left(\alpha, \frac{S}{Y}\right) \therefore$ $[1-F(\alpha)]=F\left(\frac{S}{Y}\right)$ la del trabajo, tal que: $1=[\alpha,(1-\alpha)] ;\{\alpha \mid 0 \leq \alpha<1\}$. Al diferenciar la ecuación (10) e insertar las definiciones precedentes, emana la función de distribución del crecimiento relativo de $(Y)$ :

(11) $\lambda=f[\alpha \dot{r},(1-\alpha) \dot{s}]$

Donde $\left(\lambda, \dot{r}^{\prime} s^{\prime}\right)$ son las tasas de crecimiento de $(Y, R, S)$ respectivamente. Si $\lambda=0$, resulta:

(12) $\frac{\stackrel{s}{\dot{r}}}{\mathrm{r}}=\left|-\frac{\alpha}{1-\alpha}\right|$

Las barras (II) indican |valor absoluto|. Sea $\pi=\frac{s}{\dot{s}} \therefore \pi=\frac{\alpha}{1-\alpha}$ la elasticidad-de-distribución, y:

(13) $\alpha=\frac{\pi}{1+\pi}$

Si ś $=$ ŕ $\pi$, por la ecuación (11):

(14) $\lambda=f[\alpha,(1-\alpha) \pi] \hat{r}$

$\mathrm{y}$ al insertar $\pi$ :

(15) $\lambda=2 f(\alpha \dot{r})$

32

Daniel Villalobos Céspedes

(c)

Revista Economía y Sociedad by Universidad Nacional is licensed under a CreativeCommons Reconocimiento-NoComercial- 
Equivalente de la función (11).

Para computar el ingreso de $(K, T)$ se multiplica por $(Y)$ ambos lados de la ecuación (11):

(16) $\lambda Y=[\alpha \dot{r},(1-\alpha)$ ś $] Y$

Sea $\lambda Y=\Delta Y$ el cambio absoluto en $(Y)$ tal que el cambio en $R$ es:

(17) $\Delta R=\alpha \dot{r} Y$

y el cambio en $S$ es:

(18) $\Delta S=(1-\alpha)$ ś $Y$

Al agregar las ecuaciones (17) y (18) resulta $\Delta Y=\Delta R+\Delta S$. En cada instante el ingreso del capital es $R+\Delta R=(1+$ r $) \alpha Y$ y sea $R_{t}=R_{t-1}+\Delta R$, donde $t$ denota un instante dado, para que:

(19) $R_{t}=(1+\stackrel{r}{r}) \alpha Y_{t-1}$

y para $T$ :

(20) $S_{t}=(1+\dot{s})(1-\alpha) Y_{t-1}$

Las ecuaciones (19) y (20) computan el crecimiento del ingreso del capital y del trabajo respectivamente.

\section{La ruta de la distribución del ingreso}

La razón entre las formas de ingreso define la ruta-de-distribución de $(Y)$ :

(21) $v=\frac{R}{S}$

y se reescribe la ecuación (10) como:

(22) $Y=F[(v, 1) S]$

Dado $\left[\frac{S}{Y}=(1-\alpha)\right]$, se deduce: 
(23) $v=\frac{\alpha}{1-\alpha}$

Al equiparar esta ecuación con la (12) resulta $v=\pi$; la ruta-de-distribución $(v)$ interseca la curva $(Y)$ y $(\hat{r}, \mathbf{s})$ inducirían oscilaciones de $v$. Si $\left(v \neq \pi \therefore \frac{R}{S} \neq \frac{s}{\hat{r}}\right)$ se revela divergencia en la participación de $(K, T)$ en la producción de $(Y)$ e inequidad en la distribución.

Al diferenciar la ecuación (21) se evidencia la influencia de (ŕ, ś) en la divergencia y la equidad al afectar la dirección de $v$ :

(24) $u^{\prime}=\dot{r}-s^{\prime}$

y al reemplazar ś $=$ ŕ $\pi:$

(25) ú $=(1-\pi) \dot{r}$

Si $[(s ́=0 ; \pi=0) \therefore$ v́= ́́], el salario se estanca y el capital podría acopiar parte de la productividad del trabajo. Al insertar la ecuación previa en la función (15):

(26) $\lambda=2 f\left(\alpha \frac{\dot{v}}{1-\pi}\right)$

Si ś $=0 ; \pi=0$, la curva $\lambda$ es quebrada e indica divergencia con inequidad vertical:

(27) $\lambda=2(\alpha u ́)$

La curva $(\lambda)$ converge con (ŕ) y denota divergencia con inequidad vertical.

\section{La función compuesta del crecimiento económico}

En el apartado anterior, el análisis del fenómeno convergencia-divergencia es aún limitado. La razón capital/ingreso y la tasa de retorno per se o conjugadas son insuficientes, como lo es también la razón capital/trabajo toda vez que la evolución de su valor influye en los costos de producción $(C)$ y en $(Y)$. Sea $(r)^{7}$ el retorno medio en cada instante:

(28) $\mathrm{r}=\frac{R}{K}$

${ }^{7}$ Aquí (r) difiere de (r) en Piketty (2014).

34

Daniel Villalobos Céspedes

(c) (i) (2) (2)

Revista Economía y Sociedad by Universidad Nacional is licensed under a CreativeCommons Reconocimiento-NoComercial- 
y sea (s) el salario medio:

(29) $s=\frac{S}{T}$

tal que, por la función (10):

(30) $Y=F(\mathrm{r} K, \mathrm{~s} T)$

Donde $(s T)$ identifica el ingreso del trabajo con el costo del trabajo, mientras $(r K)$ mide solo el ingreso del capital.

De la función (30), $1=F\left(\frac{K}{Y}, \frac{T}{Y}\right)$ y por la ecuación (2): $1=F[(\beta,(1-\beta)]$ donde $\beta$ computa la contribución relativa del capital en la producción. Al diferenciar la función (30) resulta $\lambda Y=$ $f[(\dot{\mathrm{r}}, k) \mathrm{r} K ;(\dot{\mathrm{s}}, n) \mathrm{s} T] \therefore \lambda=f\left[(\dot{\mathrm{r}}, k) \mathrm{r} \frac{K}{Y} ;(\dot{\mathrm{s}}, n) \mathrm{s} \frac{T}{Y}\right]$ para que:

(31) $\lambda=f[(\dot{\mathrm{r}}, k) \mathrm{r} ß ;(\dot{\mathrm{s}}, n) \mathrm{s}(1-ß)]$

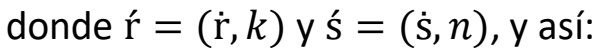

(32) $\pi=\left(\frac{\dot{\mathrm{s}}, n}{\dot{\mathrm{r}}, k}\right)$

La elasticidad-de-distribución se mide por la elasticidad-retorno del ingreso medio del trabajo $\left(\varepsilon=\frac{\dot{s}}{\dot{\mathrm{r}}}\right)$ y la elasticidad-de-composición de los recursos $\left(\mu=\frac{n}{k}\right)$. Si $\lambda=0$, de la función (31):

(33) $\left(\frac{\dot{\mathrm{s}}, n}{\dot{\mathrm{r}}, k}\right) \frac{\mathrm{s}}{\mathrm{r}}=\left|-\frac{\beta}{1-\beta}\right|$

La contribución de los recursos en la producción la determina ( $\mu$ ) mientras que la distribución la establece $(\dot{s}, \dot{r})$. De la ecuación (33), $ß=\frac{(\dot{s}, n) s}{(\dot{r}, k) r ;(\dot{s}, n) s}$ es la pendiente de la curva de produccióndistribución $(Y)$, tangente a $\left(\frac{\mathrm{r}}{\mathrm{s}}\right)$. Si $[(\dot{\mathrm{s}}, \dot{\mathrm{r}})=0 ; n=\mu k]$ ocurre $\beta=\frac{\mu \mathrm{s}}{\mathrm{r}+\mu \mathrm{s}^{\prime}}$, y si $[\mu=1, k=$ $n ;(\dot{\mathrm{s}}, \dot{\mathrm{r}})=0$ ] acaece $\beta=\frac{\mathrm{s}}{\mathrm{r}+\mathrm{s}}$. Mientras $(\mu)$ opera como una fuerza estructural que propulsa la convergencia y refuerza la divergencia en cuanto contribuye en la definición de $\pi$, $\varepsilon$ es una fuerza esencialmente sociopolítica que media la distribución. 
Por la ecuación (21):

(34) $v=\frac{\mathrm{r} K}{\mathrm{~s}} \frac{K}{T}$

Sea $\left(\omega=\frac{\mathrm{r}}{\mathrm{s}}\right)$ la frontera-de-distribución de los ingresos medios de $(K, T)$ y $\left(\mathrm{J}=\frac{K}{T}\right)$ el rayo capital/trabajo o composición física, para que:

(35) $v=\omega \mathrm{J}$

Al substituir $\pi$ y $\omega$ en la ecuación (33) resulta:

(36) $\pi=\frac{\beta}{1-\beta} \omega$

y por la ecuación (32), $\left[\dot{\mathrm{s}}, n=\frac{\beta}{1-\beta} \omega(\dot{\mathrm{r}}, k)\right]$, y dado $\omega=\frac{r}{s}$, se redefine la función (31):

(37) $\lambda=2 f[(\dot{r}, k) r ß]$

La dicotomía contribución-participación de los recursos en la productividad, revela el fenómeno convergencia-divergencia.

Dilucidar la influencia de $(\mathrm{J})$ en la producción y distribución de $(Y)$ implica comprender dos fuerzas opuestas; $K=\mathrm{J} T$ y $T=\frac{1}{\mathrm{~J}} K$. Al reemplazar $K=\mathrm{J} T$ en la función (30) se deduce $Y=$ $F(\mathrm{rJ} T, \mathrm{~s} T) \therefore Y=F(\mathrm{rJ}, \mathrm{s}) T$ y sea $y_{T}=\frac{Y}{T}$ el producto medio del trabajo, tal que:

(38) $y_{T}=F(\mathrm{rJ}, \mathrm{s})$

El producto medio por unidad adicional de trabajo permanece igual si, dada la viabilidad tecnológica y $(r, s)$, aumenta en la misma proporción la inversión en capital. Al substituir $T=\frac{1}{\mathrm{~J}} K$ en la función (30) resulta $Y=F\left(\mathrm{r} K, \frac{1}{\mathrm{~J}} \mathrm{~s} K\right) \therefore Y=F\left(\mathrm{r}, \mathrm{s} \frac{1}{\mathrm{~J}}\right) K$, y sea $y_{K}=\frac{Y}{K}$ el producto medio del capital, para que:

(39) $y_{K}=F\left(\mathrm{r}, \mathrm{s} \frac{1}{\mathrm{~J}}\right)$

36 
El producto medio por unidad adicional de capital permanece igual si, dada la viabilidad tecnológica y $(r, s)$, el trabajo aumenta en la misma proporción.

La razón entre las ecuaciones (38) y (39) destaca $\left[\mathrm{J}=\frac{y_{T}}{y_{K}}=\frac{F(\mathrm{r}, \mathrm{s})}{F\left(\mathrm{r}, \mathrm{s}_{\mathrm{J}}^{-}\right)}\right]$según productividad media de los recursos. Las tasas netas de crecimiento de $\left(\mathrm{J}, y_{T}, y_{K}\right)$ se deducen al diferenciar $\mathrm{J}=\frac{\mathrm{K}}{\mathrm{T}} \mathrm{y}$ las ecuaciones previas, de manera que:

(40) $j=k-n$

(41) $\dot{y}_{T}=\left|\lambda_{T}-n\right|$

(42) $\dot{y}_{K}=\left|\lambda_{K}-k\right|$

Al equiparar las ecuaciones (41) y (42) resulta $\left(k-n=\lambda_{K}-\lambda_{T}\right)$ y surge:

(43) $j=\lambda_{K}-\lambda_{T}$

donde $(j$ ) explica la convergencia-divergencia según dinámica de productividad del capital y del trabajo; si $\lambda=\lambda_{K}+\lambda_{T}$ entonces $\lambda=(j+1) \lambda_{T}$ y $\lambda=(j+1) \lambda_{K}$. Cambios en ( J ) no necesariamente motivan variaciones en $\left(\lambda_{T}, \lambda_{K}\right)$, pero sí $\left(\mathrm{J}=\frac{\mathrm{K}}{\mathrm{T}} \neq \frac{\lambda_{T}}{\lambda_{K}}\right)$ explicaría oscilaciones de $v$ en torno a ( $\mathrm{J}$ ); lo mismo que por la ecuación (33), si no se satisface a la función (31).

Por la derivada de la ecuación (34) se evalúa la dirección del cambio en $v$ :

(44) $\dot{v}=(\dot{\mathrm{r}}-\dot{\mathrm{s}})+(k-n)$

Si $(\dot{\mathrm{s}}=0, \mu=1)$, ocurre $\dot{v}=\dot{\mathrm{r}}$ y la ruta de $v$ tiende hacia la izquierda respecto de $\mathrm{J}$, describiendo un movimiento ascendente a lo largo de la curva $(Y)$ que delinea convergencia y divergencia con inequidad. Si $(\dot{r}, \dot{\mathrm{s}})=0$ entonces $\hat{v}=j$ y ocurre convergencia y divergencia con equidad. $(j)$ engendra la posibilidad de la divergencia mientras $(\dot{r}, \dot{\mathrm{s}})$ la de equidad; la brecha $(v, \mathrm{~J})$ revelaría divergencia en la distribución de la productividad. La dicotomía contribución-participación del capital en la productividad se mide por la función (31): $\lambda_{K}=(\dot{\mathrm{r}}, k) \mathrm{r} \beta \therefore \mathrm{r} \beta=\frac{\lambda_{K}}{(\dot{\mathrm{r}}, k)}$. Si el cambio en $(R)$ equivale al cambio en la productividad de $(K)$, entonces: $\lambda_{K} Y_{t-1}=(\dot{\mathrm{r}}, k) \mathrm{r} \beta Y_{t-1} \therefore \frac{\lambda_{K}}{(\dot{\mathrm{r}}, k)}=\frac{R_{t-1}}{Y_{t-1}} \therefore$ $\alpha=\frac{\lambda_{K}}{(\dot{r}, k)} \therefore$

(45) $\alpha=\mathrm{r} \beta=\frac{\lambda_{K}}{(\dot{r}, k)}$ 
$Y \alpha$ mide el ingreso relativo del capital respecto de los cambios en su productividad marginal.

Por la ecuación (43) $\lambda_{K}=(\dot{\mathrm{r}}, k) \mathrm{r} \beta=j+(1-\beta)(\dot{\mathrm{s}}, n) \mathrm{s}$ y al reemplazar en la (31) y dado $\dot{\mathrm{s}}, n=$ $\frac{\beta}{1-\beta} \frac{\mathrm{r}}{\mathrm{s}}(\dot{\mathrm{r}}, k)$, resulta:

(46) $\lambda=f[2(\dot{\mathrm{r}}, k) \mathrm{r} ß+j]$

$Y(j)$ opera como factor residual en contraste con $\lambda$-efectivo según funciones (31) y (37), y perturba la convergencia $\beta$ y la divergencia con equidad $\alpha$. Así, el crecimiento del producto desplazamiento de la curva $(Y)$ - depende de la elasticidad-de-composición de los recursos y de su productividad, mientras que la distribución del producto -movimiento sobre la curva $(Y)$ - de la elasticidad-retorno del salario. A efectos de mostrar la dinámica convergencia-divergencia en los procesos de producción y distribución hasta aquí estudiada, se desarrolla un ejercicio hipotético provisto en el anexo de esta investigación.

\section{Costo de producción y valor de producción}

Para comprender y entender mejor la dinámica del crecimiento económico, se analiza aquí la función de costos:

(47) $C=F(\rho K, \mathrm{~s} T)$

donde $\rho$ es el precio medio del capital. Esa función describe la participación de los recursos en los costos de producción $(C): 1=F\left(\rho \frac{K}{C}, \mathrm{~s} \frac{T}{C}\right)$ y sea $\beta=\frac{K}{C} ;\{\beta \mid 0 \leq \beta<1\}$ la participación de $(K)$ en $(C)$, tal que $1=F\left(\beta, \frac{T}{C}\right) \therefore[1-F(\beta)]=F\left(\frac{T}{C}\right)$ la participación de $(T)$ en $(C)$. Al diferenciar la ecuación (47) e insertar esas definiciones resulta la función de la tasa de crecimiento de $(C)$ : (48) ć $=f[(\dot{\rho}, k) \rho \beta ;(\dot{\mathrm{s}}, n) \mathrm{s}(1-\beta)]$

Si ć $=0$, entonces:

(49) $\left(\frac{\dot{s}, n}{\rho, k}\right) \frac{S}{\rho}=\left|-\frac{\beta}{1-\beta}\right|$

Se computa $\beta$ como:

(50) $\beta=\left|-\frac{(\dot{\mathrm{s}}, n) \mathrm{s}}{(\dot{\rho}, k) \rho ;(\dot{\mathrm{s}}, n) \mathrm{s}}\right|$

38

Daniel Villalobos Céspedes

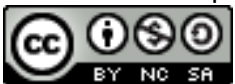

Revista Economía y Sociedad by Universidad Nacional is licensed under a CreativeCommons Reconocimiento-NoComercial- 
Sea $\eta=\left(\frac{\dot{s}, n}{\rho, k}\right)$ la elasticidad-de-substitución de $(T)$ por $(K)$, medida por la elasticidad-decomposición física $(\mu)$ y la elasticidad-de-composición precio $\left(\varnothing=\frac{\dot{s}}{\dot{\rho}}\right)$ de los recursos y se reescribe la ecuación (49):

(51) $\eta=\frac{\beta}{1-\beta} \frac{\rho}{s}$

Dado $s(\dot{\mathrm{s}}, n)=(\dot{\rho}, k) \rho \frac{\beta}{1-\beta}$, al reemplazar en la ecuación (48) resulta:

(52) $c ́=2 f[(\rho, k) \rho \beta]$

Al equiparar las ecuaciones (33) y (49) tal que $(\dot{\rho}, k)=(\dot{r}, k) \frac{\mathrm{r}}{\rho}$ y al reemplazar en la función anterior, se obtiene:

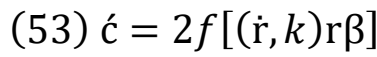

Al equiparar esta función con la (37) resulta $[\lambda=c ́ c \beta=\beta ; \mathrm{r}=\rho]$. Además, $\frac{\rho}{\mathrm{r}}=\left(\frac{\dot{\mathrm{r}}, k}{\dot{\rho}, k}\right)$ destaca el retorno respecto del costo del capital: $(\lambda-\dot{c})=2 f[(\dot{r}, k) \mathrm{r} \beta]-2 f[(\dot{\rho}, k) \rho \beta] \therefore$

(54) $\mathrm{r} \beta=\left(\frac{\dot{\rho}, k}{\dot{\mathrm{r}}, k}\right) \rho \beta$

De esa ecuación $\mathrm{r}=\left(\frac{\dot{\rho}, k}{\dot{\mathrm{r}}, k}\right) \rho \frac{\mathrm{Y}}{C}$ y sea $c=\frac{C}{Y}$ el costo medio de producción, para que:

(55) $\mathrm{r}=\left(\frac{\dot{\rho}, k}{\dot{\mathrm{r}}, k}\right) \frac{\rho}{c}$

Ceteris paribus, el retorno medio varía inversamente a los cambios en el costo medio de producción, pero en razón directa a la variación del precio medio del capital y de la inversión en capital.

Aún más elegante: De la ecuación (54) $\alpha=\left(\frac{\dot{\rho}, k}{\dot{r}, k}\right) \rho \beta$ y sea $\grave{\alpha}=\rho \beta$ para que $\alpha=\left(\frac{\dot{\rho}, k}{\dot{r}, k}\right) \grave{\alpha}$ y se establece que la influencia de $(K)$ en el valor de $(Y)$ depende del efecto de $\rho$ en $\dot{r}$; si $k=0$, resulta $\alpha=\frac{\dot{\rho}}{\dot{\mathrm{r}}} \grave{\alpha}$. Al insertar la ecuación (54) en la función (31) se deduce la (48); la función de crecimiento 
de $(Y)$ se distancia de la función de crecimiento de $(C)$ debido a la magnitud de $(R)$. Dado ŕs $(\dot{r}, K)$ resulta:

(56) $\dot{\mathrm{r}}=(\dot{\rho}, k) \frac{\grave{\alpha}}{\alpha}$

y se explica que las variaciones en la tasa de retorno se deben a cambios en los precios del capital y de la inversión en capital, pero también de la participación del capital en los costos de producción respecto de su participación en el producto. La definición de $(r)$ es ahora más amplia: $\mathrm{r}=\frac{R}{\rho K} \therefore R=\mathrm{r} \rho K$ y al diferenciar $\dot{\mathrm{r}}=(\dot{\mathrm{r}}, \dot{\mathrm{p}}, \mathrm{k})$ y $(\mathrm{k}=0, \dot{\mathrm{p}}>0)$ podría inducir un alza proporcional en $\dot{r}^{8}$

Al reemplazar esa definición previa en la función (31) se obtiene:

(57) $\lambda=f[(\dot{\mathrm{r}}, \dot{\rho}, k) \mathrm{r} ß ;(\dot{\mathrm{s}}, n) \mathrm{s}(1-\beta)]$

Si $\lambda=0$, ocurre:

(58) $\left(\frac{\dot{\mathrm{s}}, n}{\dot{\mathrm{r}}, \dot{\rho}, k}\right) \frac{\mathrm{s}}{\mathrm{r}}=\left|-\frac{\beta}{1-\beta}\right|$

y se computa $\beta$ como:

(59) $\beta=\left|-\frac{(\dot{\mathrm{s}}, n) \mathrm{s}}{(\dot{\mathrm{r}}, \dot{\rho}, k) \mathrm{r} ;(\dot{\mathrm{s}}, n) \mathrm{s}}\right|$

De la función (47) se define el valor de la composición de los recursos en los costos de producción como:

(60) $\sigma=\frac{\rho}{\mathrm{S}} \frac{K}{\mathrm{~T}}$

y si $\mathrm{J}=\frac{K}{\mathrm{~T}}$ entonces:

(61) $\sigma=\frac{\rho}{\mathrm{S}} \mathrm{J}$

${ }^{8}$ En algunas empresas $\dot{p}=\dot{r}$ dependerá de la intensidad de la competencia en la industria y la economía. 40

Daniel Villalobos Céspedes

(c) (1) $\$$ (9)

Revista Economía y Sociedad by Universidad Nacional is licensed under a CreativeCommons Reconocimiento-NoComercial- 
y se reescribe la función (47) como $C=F(\sigma \mathrm{s} T, \mathrm{~s} T) \therefore C=F(\sigma, 1)$ sT $T$ y de modo equivalente $C=$ $F\left(\rho K, \frac{1}{\sigma} \rho K\right) \therefore C=F\left(1, \frac{1}{\sigma}\right) \rho K$. Estas definiciones explican que el costo de producción varía en proporción a los cambios en el valor de la composición de los recursos. ${ }^{9}$

Se desprende que $\frac{C}{\mathrm{~s} T}=F(\sigma, 1) ; \frac{C}{\rho K}=F\left(1, \frac{1}{\sigma}\right)$ y sea $c_{T}=\frac{C}{\mathrm{~s} T} \therefore c_{T}=F(\sigma, 1)$ el costo medio del trabajo por unidad de capital y $c_{K}=\frac{C}{\rho K} \therefore c_{K}=F\left(1, \frac{1}{\sigma}\right)$ el costo medio del capital por unidad adicional de trabajo. Al diferenciar resultan los respectivos costos marginales del trabajo y el capital: $\left[\dot{\mathrm{c}}_{T}=\dot{\mathrm{c}}_{T}-(\dot{\mathrm{s}}, n)\right]$ y $\left[\dot{\mathrm{c}}_{K}=\dot{\mathrm{c}}_{K}-(\dot{\rho}, k)\right]$, o bien de la función (48): el costo marginal del capital es $\left[\hat{c}_{K}=(\dot{\rho}, k) \rho \beta \therefore \grave{\alpha}=\rho \beta=\frac{\dot{c}_{K}}{(\dot{\rho}, k)}\right.$ y el costo marginal del trabajo es $\dot{c}_{T}=(\dot{\mathrm{s}}, n) \mathrm{s}(1-$ $\beta) \therefore \frac{\hat{c}_{T}}{(\dot{s}, n)}=\mathrm{s}(1-\beta)$. Al equiparar la contribución relativa del trabajo al costo con la contribución relativa al ingreso se deriva $\frac{\dot{c}_{T}}{(\dot{s}, n)}=\frac{\lambda_{T}}{(\dot{s}, n)} \therefore \dot{c}_{T}=\lambda_{T}$ y podría diferir de $\lambda_{T}$, mientras que para el capital $\frac{\dot{c}_{K}}{(\hat{\rho}, k)}=\frac{\lambda_{K}}{(\dot{r}, \dot{\rho}, k)}=\dot{c}_{K}=\frac{(\dot{\rho}, k)}{(\dot{\mathrm{r}}, \hat{\rho}, k)} \lambda_{K}$ que podría ser diferente de $\lambda_{K}$. La razón $\frac{c_{T}}{c_{K}}=\frac{F\left(\frac{\rho}{\mathrm{s}} \mathrm{J}, 1\right)}{F\left(1, \frac{\hat{\rho}}{\rho} \frac{1}{\mathrm{~J}}\right)}=\sigma \mathrm{y}$ al equiparar con el resultado de la razón entre las ecuaciones (38) y (39) $\frac{y_{T}}{y_{K}}=\frac{c_{T}}{c_{K}} \therefore \frac{F(\mathrm{r}, \mathrm{s})}{F\left(\mathrm{r}, \mathrm{s}_{\mathrm{J}}^{-}\right)}=$ $\frac{F\left(\frac{\rho}{\mathrm{s}} \mathrm{J}, 1\right)}{F\left(1, \frac{\mathrm{s}}{\rho} \frac{1}{\mathrm{~J}}\right)}$ ocurre $\mathrm{J}=\sigma$ y $\rho=\mathrm{s}$. Al equiparar las ecuaciones (34) y (60) resulta $v=\sigma \therefore \mathrm{r}=\rho$ y la ganancia es nula; la composición física de los recursos podría no cambiar mientras su valor fluctúa, y viceversa.

La influencia de $(K, T)$ en J se mide según su productividad media y costo medio. Si el producto medio del trabajo según ecuación (39) equivale al costo medio del trabajo, entonces $y_{T}=c_{T} \therefore$ $F(r \mathrm{~J}, s)=F\left(\frac{\rho}{s} \mathrm{~J}, 1\right)$ tal que:

(62) $\mathrm{r}=\frac{\rho}{\mathrm{s}}+\frac{1-\mathrm{s}}{\mathrm{J}}$

Y sea $\tilde{\omega}=\frac{\rho}{s}$ la línea-de-distribución del costo medio de los recursos. Esa ecuación explica la influencia de los costos medios de los recursos ceteris paribus en el retorno medio a cada instante

\footnotetext{
${ }^{9}$ Las ecuaciones (60) y (61) asemejan la composición orgánica del capital de Marx: "En cualquier momento dado, la composición orgánica del capital depende de dos circunstancias: en primer lugar, de la relación técnica entre la fuerza de trabajo empleada y la masa de medios de producción; y en segundo término, del precio de esos medios de producción" (Marx, 1986, p. 195).
} 
a causa del trabajo por unidad de capital. Para el caso del capital; $y_{K}=c_{K} \therefore F\left(r, s \frac{1}{\mathrm{~J}}\right)=$ $F\left(1, \frac{\mathrm{s}}{\rho} \frac{1}{\mathrm{~J}}\right)$ para que:

(63) $\mathrm{r}=1+\frac{1-\rho}{\mathrm{J}} \frac{\mathrm{s}}{\rho}$

y se explica la influencia de los costos medio de los recursos ceteris paribus en el retorno medio a cada instante, por parte del capital por unidad de trabajo. Esta dinámica del costo y valor de producción y el retorno se ilustra en la Figura $\underline{1}$.

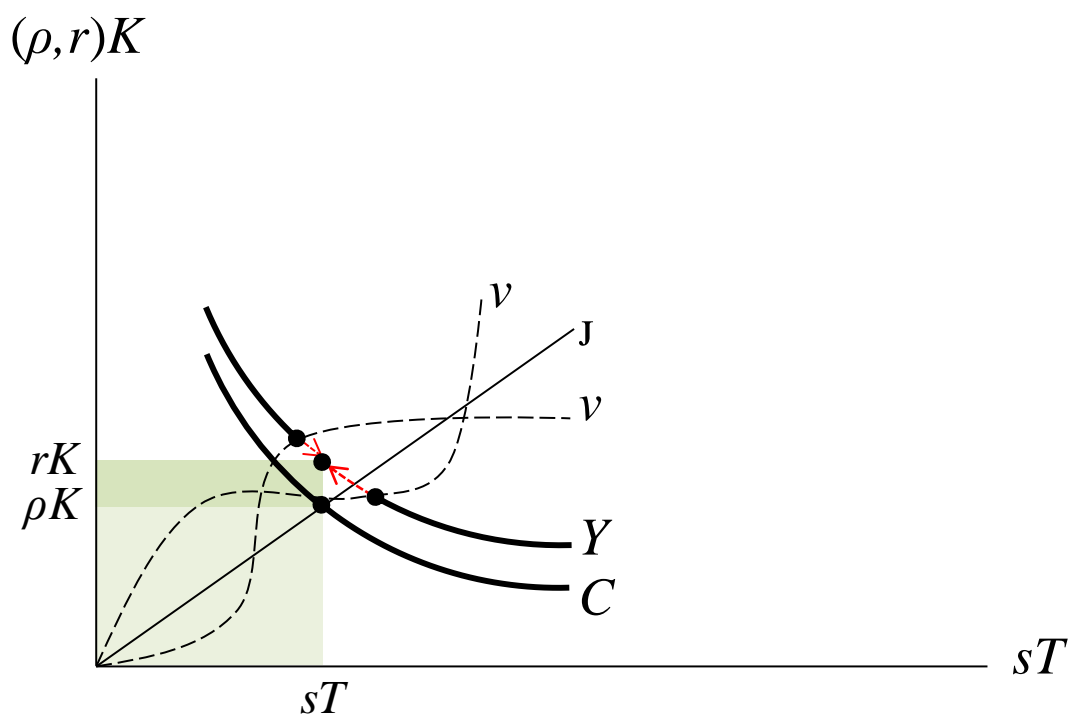

Figura 1. Convergencia y divergencia. Oscilación de $\boldsymbol{v}, \boldsymbol{\sigma}$ en torno a J. Fuente: elaboración propia.

Conviene cerrar este apartado anotando que la ecuación (31) admite describir la producción con proporciones fijas de los recursos y crecimiento económico nulo o proporcional. Por la ecuación (33) si, ceteris paribus, $[(\dot{\mathrm{r}}, k)=0 ; \beta=1 ; \mu=\infty]$ y $[(\dot{\mathrm{s}}, n)=0 ; \beta=0 ; \mu=0]$; y en ambos sucesos $\lambda=0$. Si $\left[(k=n) ; ß=\left|\frac{\mathrm{s}}{\mathrm{r}, \mathrm{s}}\right| ; \mu=1\right]$ y dado $(\dot{\mathrm{s}}, \dot{\mathrm{r}})=0$ se describe crecimiento constante a escala.

\section{Ganancia y tasa de ganancia}

En el proceso de producción, el retorno no es un atributo de $K$ solamente, entre otras circunstancias, de T. Esto es, $(K, T)$ operan conjuntamente en la formación de $(R)$ por medio de la ganancia (G). Mientras (G) es el resultado de las fuerzas del mercado de bienes y servicios, la 42 
composición media de los recursos y la competencia, $(R)$ lo es, además, de las fuerzas del mercado financiero y de las políticas económicas globales. Si $\mathrm{R} R \cong G$, la función compuesta equivalente de (30) es:

(64) $Y=F(G, \mathrm{~s} T)$

La tasa de variación de la ganancia constituye un componente de la tasa de crecimiento del valor de la producción. Sea la función de ganancia:

(65) $G=F(\rho K, \mathrm{~s} T)$

y la participación de los recursos es $\left[1=F\left(\frac{K}{G}, \frac{T}{G}\right)\right]$. Sea $\left[B=\frac{K}{G},\{B:(0 \leq B<1)\}\right]$ la contribución del capital en la creación de la ganancia, para que $\left[1=F\left(\beta, \frac{T}{G}\right)\right]$ tal que $[1-F(\beta)]=F\left(\frac{T}{G}\right)$ se la contribución del trabajo.

Al diferenciar la ecuación (65) resulta:

(66) $\mathrm{g}=f[(\dot{\rho}, k) \rho ß,(\dot{\mathrm{s}}, n)(1-\beta) \mathrm{s}]$

y ǵ es la tasa de variación de la ganancia. Si ǵ = 0 ocurre:

(67) $\left(\frac{\dot{\mathrm{s}}, n}{\dot{\rho}, k}\right) \frac{\mathrm{s}}{\rho}=\left|-\frac{\beta}{1-\beta}\right|$

De donde se puede calcular el valor de $\beta$. De la ecuación $(67)(\dot{\mathrm{s}}, n)=(\rho, k) \frac{\beta}{1-\beta} \frac{\rho}{\mathrm{s}}$, y al insertar esta definición en la ecuación (66) resulta:

(68) $\dot{g}=2 f[(\rho, k) \rho ß]$

Si $\left(c^{\prime}+g\right)=2 f[(\dot{\rho}, k) \rho \beta]+2 f[(\dot{\rho}, k) \rho ß]=2 f[(\dot{\rho}, k) \rho(\beta, \beta)]$ y si $\beta=(\beta, \beta)$ entonces (ć + $\mathrm{g})=2 f[(\dot{\rho}, k) \rho ß]$ y se extrae de las funciones (57), (52) y (67) el componente financiero ( $\dot{\mathrm{r}})$ del crecimiento del valor de producción: $[\lambda-(\dot{c}+\dot{g})=2 f[(\dot{r}, \dot{\rho}, k) r ß]-2 f[(\dot{\rho}, k) \rho ß]]$ tal que:

(69) $\dot{r}=-\left[\frac{r-\rho}{r}(\rho, k)\right]$

y se computa la propensión de ǵ a decrecer al insertar la ecuación anterior en (68) y dado ( $\dot{r}=\dot{r}$ k): 
(70) $\mathrm{g}=-2 f\left[\left(\frac{\mathrm{r}}{\mathrm{r}-\rho}\right)(\dot{\mathrm{r}}-k) \rho ß\right]$

ceteris paribus $\dot{r}$ podría incidir en la tendencia decreciente de la tasa de ganancia en una economía, lo que podría atribuirse a la substitución de inversión productiva por inversión financiera; si $(\dot{r}=0)$ ceteris paribus un aumento en $(k)$ induce un alza en $g$, y viceversa:

(71) $\mathrm{g}=2 f\left[\left(\frac{\mathrm{r}}{\mathrm{r}-\rho}\right) k \rho \beta\right]$

Si $(k=0)$ ceteris paribus un aumento en ŕ estimula una caída en ǵ pero una reducción en ŕ contrarresta la caída en ǵ:

(72) $\dot{\mathrm{g}}=-2 f\left[\left(\frac{\mathrm{r}}{\mathrm{r}-\rho}\right) \hat{\mathrm{r}} \rho ß\right]$

Para visualizar los efectos de $(\dot{s}, n, \mathrm{~s})$ en ǵ, no revelados por la función (70), se requiere operar con las funciones (48) y (57).

\section{Conclusiones y posibles líneas de investigación}

En esta investigación se inició con las críticas que algunos destacados estudios propinaron a la obra de Piketty (2014), y se planteó operacionalizar la propuesta de ese autor con el objeto de descubrir entre las relaciones conceptuales una posible explicación de su supuesto crucial: $r>g$. Se develó que la posibilidad de $r>g$ no es útil para evidenciar las causas fundamentales de la convergencia y la divergencia tal como se lo propuso Piketty (2014). Al cuestionar ese supuesto crucial con mayor refinamiento matemático ${ }^{10}$, según sugirió Cobb y Douglas (1928), resulta un modelo de crecimiento económico que propone que la dicotomía convergencia-divergencia es inherente a los procesos de producción y distribución, y no exclusivo de una economía de mercado como concluyó Piketty (2014). La divergencia no es sinónimo de inequidad, como la convergencia no lo es de equidad.

La composición física y de valor de los recursos determina la convergencia en los procesos de producción. No obstante, medida según la productividad de los recursos podría distorsionar la

\footnotetext{
10 Piketty (2014) evadió este proceder argumentando que no es el propósito de las investigaciones en las ciencias sociales, debido a que pueden substituir un debate abierto, democrático en el cual todas las opiniones estén representadas (p.571). Empero, esta investigación parte del criterio de que el supuesto crucial de ese autor es dudoso y los resultados sospechosos (Solow) por burda la formulación matemática, lo que exigió un análisis riguroso pertinente, quizá no exhaustivo, para un debate menos abierto, menos democrático, que incita principalmente a la crítica social que ha de residir en la ciencia económica.
}

44

Daniel Villalobos Céspedes

(c) (1) 8 (2)

Revista Economía y Sociedad by Universidad Nacional is licensed under a CreativeCommons Reconocimiento-NoComercial- 
divergencia, relativa a la distribución del crecimiento del producto en la forma de ingreso. El modelo aquí propuesto podría responder a la pregunta de Cobb y Douglas (1928) referida en la introducción: Naturalmente, "los procesos de distribución son...modelados por los procesos de producción de valor" (Cobb \& Douglas, 1928, pp. 139-140). Las preocupaciones de Harrod (1939) y (Harrod, 1960) en relación a cómo operan las fuerzas con influjo en la tendencia del crecimiento y en el cambio sostenido en la distribución del ingreso también podrían haber recibido respuesta en esta investigación; el ejercicio hipotético remitido al apéndice podría dar luz acerca de disyuntivas. Si en una economía el salario real se estanca o crece menos que la productividad media del trabajo (Solow, 2014), ceteris paribus, ocurre divergencia con inequidad.

El modelo planteado admite la necesidad de políticas públicas orientadas a corregir las desviaciones de la natural convergencia-divergencia que caracteriza los procesos de produccióndistribución. Si bien no existe una "solución correcta", "un impuesto progresivo al capital" (Piketty, 2014, p. 572), así como otras fuerzas "centrífugas y centrípetas" (Stiglitz, 2015), tales como el control demográfico y del poder de mercado y la explotación, podrían contribuir a direccionar la divergencia con inequidad. Los procesos de distribución engendran, inexorablemente, la acumulación de riqueza por parte de algunas personas, entre las cuales el sector capitalista aventaja al trabajador tal como destacan Solow (2014) y Mankiw (2015) y a pesar de Galbraith (2014). Futuras investigaciones han de mejorar el modelo aquí propuesto para valorar la influencia de esas variables y otras propias de la política fiscal, monetaria, social y comercial de los gobiernos, en las distorsiones del fenómeno en cuestión.

\section{Referencias}

Arrow, J. K., Chenery, B. H., Minhas, S. B., \& Solow, M. R. (1961). Capital-Labor Substitution and Economic Efficiency. The Review of Economics and Statistics, 3(43), 225-250. doi: https://doi.org/10.2307/1927286

Cobb, W. C., \& Douglas, H. P. (1928). A Theory of Production. The American Economic Review, 18(1), 139-165. Recuperado de https://www.jstor.org/stable/1811556?origin=JSTORpdf\&seq=1\#page scan tab contents

Domar, E. D. (1946). Capital Expansion, Rate of Growth, and Employment. Econometrica, 2(14), 137-147. doi: https://doi.org/10.2307/1905364

Domar, E. D. (1953). Depreciation, Replacement and Growth. The Economic Journal, 249 (63), 132. doi: https://doi.org/10.2307/2226748 
Galbraith, J. K. (2014). Kapital for the Twenty-First Century? En Dissent Magazine. Recuperado de https://www.dissentmagazine.org/article/kapital-for-the-twenty-first-century

Goldhammerr, A. (2017). The Piketty Fenomenon. En J. B. Heather Boushey, After Piketty, The Agenda for Economics and Inequality (First ed., 36-59). London, England: Harvard University Press. doi: https://doi.org/10.4159/9780674978195-002

Harrod, R. F. (1939). An Essay in Dynamic Theory. The Economic Journal, 193(49), 14-33. doi: https://doi.org/10.2307/2225181

Harrod, R. F. (1960). Second Essay in Dynamic Theory. The Economic Journal, 278 (70), 277-293. doi: https://doi.org/10.2307/2228728

Krugman, P. (2014). Why we're in a New Gilded Age. En The New York Review of Books. Recuperado de https://www.nybooks.com/articles/2014/05/08/thomas-piketty-newgilded-age/

Krugman, P. (2 de agosto 2015). The Economics of Inequality by Thomas Piketty. The New York Times. Recuperado de https://www.nytimes.com/2015/08/03/books/review-theeconomics-of-inequality-by-thomas-piketty.html

Mankiw, N. G. (2015). Yes, r > g. So What? American Economic Review, 5(105), 43-47. doi: https://doi.org/10.1257/aer.p20151059

Marx, K. (1984). El Capital. La tasa de plusvalor (Tomo I, Volumen I): El proceso de producción del capital (14ºd., 255-269). México: Siglo XXI.

Marx, K. (1986). El Capital. Formación de una tasa general de ganancia (Tasa media de ganancia) y transformación de los valores mercantiles en precios de producción). En Tomo III, Vol. VI: El Proceso global de la producción capitalista (8 va ed., 195-217). México: Siglo XXI.

Naidu, S. (2017). A Political Economy take on W/Y. En H. Boushey, J. B. DeLong, \& M. Steinbaum, After Pikety. The Agenda for Economics and Inequality (First ed., 120-154. London, England: Harvard University Press. doi: https://doi.org/10.4159/9780674978195-006

Piketty, T. (2014). Capital in the Twenty-First Century (First ed., 1-685). London: The Belknap Press of Harvard University Press. Doi: https://doi.org/10.4159/9780674369542

46

Daniel Villalobos Céspedes

(c) (1) 8 (9)

Revista Economía y Sociedad by Universidad Nacional is licensed under a CreativeCommons Reconocimiento-NoComercial- 
Piketty, T. (2017). Toward a Reconciliation between Economics and the Social Sciencies. En H. Boushey, J. B. DeLong, \& M. Steinbaum, After Piketty. The Agenda for Economics and Inequality (First ed., 628-860). London, England: Harvard University Press. doi: https://doi.org/10.4159/9780674978195-023

Raval, D. (2017). What's Wrong with Capital in the Twenty-First Century's Model? En H. Boushey, J. B. DeLong, \& M. Steinbaum, After Piketty. The Agenda for Economics and Inequality (First ed., 86-119). London, England: Harvard University Press. doi: https://doi.org/10.4159/9780674978195-005

Schuessler, J. (2014). Economist Receives Rock Star Treatment. En The New York Times. Recuperado de https://www.nytimes.com/2014/04/19/books/thomas-piketty-tours-usfor-his-new-book.html? $r=0 \&$ module=inline.

Solow, M. R. (1956). A Contribution to the Theory of Economic Growth. The Quarterly Journal of Economics, 1(70), 65-94. doi: https://doi.org/10.2307/1884513

Solow, M. R. (1957). Technical Change and the Aggregate Production Function. The Review of Economics and Statistics, 3(39), 312-320. doi: https://doi.org/10.2307/1926047

Solow, M. R. (22 de abril 2014). Thomas Piketty is Right. The New Republic. Recuperado de https://newrepublic.com/article/117429/capital-twenty-first-century-thomas-pikettyreviewed

Stiglitz, J. E. (2015). New Theoretical Perspective on the Distribution of Income and Wealth Among Individuals: Part I. The Wealth Residual. En NBER Working Paper 21189, National Bureau of Economic Research. Recuperado de http://www.nber.org/papers/w21189

Swan, T. (1956). Economic Growth and Capital Accumulation. Economic Record, 2(32), 334-361. Doi: $\underline{\text { https://doi.org/10.1111/j.1475-4932.1956.tb00434.x }}$

Villalobos, D. (2010). Capital, competencia y ganancia (1 ${ }^{\text {era }}$ ed.). Heredia: EUNA. Recuperado de https://www.euna.una.ac.cr/index.php/EUNA/catalog/book/110 


\section{Apéndice}

\section{Ejercicio hipotético: Dinámica de la convergencia-divergencia}

El propósito del siguiente ejercicio hipotético es mostrar la dinámica de la convergenciadivergencia innata a los procesos de producción y distribución según el modelo de crecimiento económico propuesto en la presente investigación; no abarca de manera explícita los apartados quinto y sexto. A efectos de facilitar su comprensión se indica el desarrollo del ejercicio el número de las funciones y ecuaciones utilizadas. Al equiparar las funciones (15) y (37) resulta $[2 f(\alpha \dot{\mathrm{r}})=2 f[(\dot{\mathrm{r}}, k) \mathrm{r} \beta]]$ y dado $(\dot{\mathrm{r}}=\dot{\mathrm{r}}, k)$ resulta $(\alpha=\mathrm{r} \beta)$. La función (37) contiene $\alpha$ dado $\pi=$ $\frac{\alpha}{1-\alpha}$ conforme a la ecuación (12), tal que por la ecuación (36) $\left[\frac{\alpha}{1-\alpha}=\frac{\beta}{1-\beta} \omega\right]$. Por la ecuación (10), $(1-\mathrm{r} \beta)=\left(\frac{S}{Y}\right) \mathrm{y}$, si persiste la equidad, $r \beta=\left(\frac{S}{Y}\right)$ para que $(1-r \beta)=r \beta$ y $[1=\mathrm{r} \beta+\mathrm{r} \beta \therefore$ $\left.\mathrm{r} \beta=\frac{1}{2}\right], \mathrm{y}\left[\alpha=r \beta=\frac{1}{2}\right] ;$ o, por la ecuación (13): $\alpha=\frac{\pi}{1+\pi}$ y en equidad $\left[\pi=\frac{\dot{s}}{\hat{\mathrm{r}}}=1\right]$ por lo que $\alpha=\frac{1}{2}$. Se computa en (15) $\lambda=2 f\left(\frac{1}{2} \dot{\mathrm{r}}\right)$ y resulta convergencia y divergencia con equidad. En contraste con Piketty, es posible calcular $\beta$, y por su medio $\alpha$, si (r) es un dato y es medible $(\dot{r}=\dot{r}, k)$.

Al suponer $(\dot{\mathrm{r}}, \dot{\mathrm{s}})=0 ; \pi=\mu$ y $\mu=\frac{\beta}{1-\beta} \omega \therefore \beta=\frac{\mu}{\omega+\mu}$ y $\omega=\frac{\mathrm{r}}{\mathrm{s}}$ tal que $\beta=\frac{\mu \mathrm{s}}{\mathrm{r}+\mu \mathrm{s}}$, la posibilidad de la divergencia con inequidad es aún notable. Sea $(\mu=1 ; r=s)$ para que $\left(\frac{\beta}{1-\beta}=1 \therefore \beta=\frac{1}{2}\right)$ o bien $\left(\beta=\frac{r}{r+r} \therefore \beta=\frac{1}{2}\right)$. Ejemplo: $r=0.3$ - esto es, $r=30 \%-$ tal que $\left(\beta=\frac{0.3}{0.3+0.3}=\frac{1}{2}\right)$ y así $\left[\alpha=r \beta \therefore \alpha=(0.3)\left(\frac{1}{2}\right)=0.15\right]$. Si $[(k=n)=0.01 ; \mu=1]$, por la función (31) se computa $\lambda=\left[(0.01)(0.3)\left(\frac{1}{2}\right) ;(0.01)\left(1-\frac{1}{2}\right)(0.3)\right] \cong 0.0015+0.0015 \cong 0.003$. Por la función (15) $[\lambda=2 f(\alpha \dot{r}) \therefore \lambda=2(0.15)(0.01)]=0.003, \quad$ y por la función (37) se obtiene $\lambda=$ $2 f\left[(0.01)(0.3)\left(\frac{1}{2}\right)\right]=0.003$. En convergencia ocurre divergencia con equidad si $[\alpha=\mathrm{r} \beta=$ $(1-ß) \mathrm{s}]$ tal que $\left[\alpha=(0.3)\left(\frac{1}{2}\right)=\left(1-\frac{1}{2}\right)(0.3)=0.15\right]$.

Además, $\left(\pi=\frac{\alpha}{1-\alpha} \therefore \pi=\frac{0.15}{1-0.15} \therefore \pi=0.1765=17.65 \%\right)$ es la fracción del crecimiento del valor de $(Y)$ que recibirá el capital y $[(1-\pi)=(1-0.1765) \cong 0.8235=82.35 \%]$ corresponde al trabajo; se refleja convergencia en la producción y divergencia con equidad en la distribución de $(Y)$. Por la función (15) $[\lambda=2(\alpha \dot{r}) \therefore \lambda=2(0.15)(\dot{\mathrm{r}}, k)]$ y dado $[\dot{\mathrm{r}}=0 ;(k=$ $n)=0.01]$ resulta $[\lambda=2(0.15)(0.01) \therefore \lambda=0.003]$, lo mismo que con las funciones (31) y (37). ¿Cómo se distribuye el crecimiento del valor de producción? La síntesis de los fenómenos convergencia y divergencia consiste en: Primero, la productividad de los recursos se evalúa como $\left[\lambda=\lambda_{K}+\lambda_{T} \therefore \lambda=0.0015+0.0015=0.003\right]$. Segundo, la distribución del producto se calcula

48

Daniel Villalobos Céspedes

(c) (i) (ㅇ)

Revista Economía y Sociedad by Universidad Nacional is licensed under a CreativeCommons Reconocimiento-NoComercial- 
como $\left[\lambda=\lambda_{K}+\lambda_{T}\right]$, donde $\left[\lambda_{K}=\pi \lambda \therefore \lambda_{K}=(0.1765)(0.003) \therefore \lambda_{K} \cong 0.0005295\right]$ y $\left[\lambda_{T}=\right.$ $\left.(1-\pi) \lambda \therefore \lambda_{T}=(0.8235)(0.003) \therefore \lambda_{T} \cong 0.002471\right]$ de manera que $\left[\lambda=\lambda_{K}+\lambda_{T} \therefore \lambda \cong\right.$ $0.0005295+0.002471 \therefore \lambda \cong 0.003]$.

La transferencia de valor de producción entre los recursos por el mecanismo de distribución se obtiene como sigue: $\left[\lambda_{K}-\lambda_{K}=0.0005295-0.0015 \cong-0.0009705\right]$ indica que el ingreso relativo del capital es menor que su productividad relativa y $\left[\lambda_{T}-\lambda_{T}=0.002471-0.0015=\right.$ $0.0009705]$ refleja que el ingreso del trabajo es mayor que su contribución relativa al producto. En la economía no se pierde nada, puesto que el producto marginal que un recurso recibe como excedente en la forma de ingreso, corresponde al producto marginal no retribuido como ingreso a otro u otros recursos.

Al insertar $\mathrm{r} K=v \mathrm{~s} T$ según ecuación (34) en la función (30) resulta $Y=F(v \mathrm{~s} T, \mathrm{~s} T) \therefore Y=$ $F(v, 1)$ s $T$, y al derivar e insertar las definiciones previas se obtiene:

$\lambda=f[(\dot{v} v) ;(v, 1)(\dot{s}, n)] \mathrm{s}(1-\beta)$

Con esa función se computa, según ejercicio anterior: $\left[0.003=[(v, 1)(0.01)](0.3)\left(\frac{1}{2}\right) \therefore\right.$ $0.003=[(v, 1)(0.01)](0.15)=0.02=(v, 1)(0.01) \therefore v=\mu=1]$ y $(\mathrm{J}=v=1)$. La ecuación (44) evalúa la magnitud y dirección del cambio en $v$, y dado $(\dot{\mathrm{r}}, \dot{\mathrm{s}})=0 ;(k=n)=0.01 \therefore j=0$, por la ecuación anterior $v=0$; es decir, $(v=\mathrm{J})$ no varían. Se comprueban estos resultados por $\frac{\lambda_{T}}{\lambda_{K}}=1$, que explica igual productividad de los recursos, acorde a la convergencia en la composición. En contraste, $\frac{\lambda_{T}}{\lambda_{K}}=\frac{0.002471}{0.0005295}=4.667$, lo que denota divergencia con equidad en la distribución de $(Y)$. El ingreso relativo del trabajo supera su productividad relativa: $\left(\lambda_{T}>\lambda_{T} \therefore 0.00261>0.0015\right)$ o bien que $\frac{\lambda_{T}}{\lambda_{T}} \therefore \frac{0.002471}{0.0015}=1.65$ veces $\mathrm{y}$ para el capital $\left(\lambda_{K}<\lambda_{K} \therefore 0.0005295<0.0015\right)$ en razón de $\frac{\lambda_{K}}{\lambda_{K}} \therefore \frac{0.0005295}{0.0015}=0.35$ veces. En una economía, la divergencia en la distribución del valor de producción podría ser congruente con la convergencia en la composición de los recursos.

Si en esa economía sucede un cambio en la viabilidad tecnológica tal que $\left(k=1 \frac{1}{5} n \therefore \mu=\frac{n}{1 \frac{1}{5} n}=\right.$ $\left.\frac{5}{6} \cong 0.8 \overline{3}\right)$ y $[(\dot{\mathrm{r}}=k, \dot{\mathrm{r}}=0) ;(\dot{\mathrm{s}}=n, \dot{\mathrm{s}}=0)] \therefore\left[(\mathrm{r}=\mathrm{s}):(\dot{\mathrm{r}}>\dot{\mathrm{s}}) \therefore \frac{\dot{\mathrm{r}}}{\mathrm{s}}=\frac{0.012}{0.01}=1.2\right]$ para que $[\beta=$ $\left.\frac{\mu \mathrm{s}}{r+\mu \mathrm{s}} \therefore \beta=\frac{\left(\frac{5}{6}\right)(0.3)}{(0.3)+\left(\frac{5}{6}\right)(0.3)} \therefore \beta \cong 0.45 \overline{45}\right] \quad$ y $\quad\left[j=\left(1 \frac{1}{5} n-n\right)=0.2 n\right] ; \quad$ ( J $) \quad$ se $\quad$ desplaza verticalmente. 
Además $\alpha=(0.3)(0.45 \overline{45}) \cong 0.1364$ es menor respecto de la situación precedente. Por la función $\quad$ (31): $\quad \lambda=[(0.012)(0.3)(0.45 \overline{45}) ;(0.01)(1-0.45 \overline{45})(0.3)] \cong 0.001636+$ $0.001636 \cong 0.003273$; idéntico resultado se obtiene con las funciones (15) y (37). La productividad neta del trabajo crece en $\left[\dot{y}_{T}=\left|\lambda_{T}-n\right| \therefore \dot{y}_{T}=|0.0015-0.01|=0.0085\right] \rightarrow$ $\left[\dot{y}_{T}=|0.001636-0.01|=0.00864\right]$ y su contribución relativa al ingreso en $\left[\frac{\lambda_{T}}{(\dot{s}, \mathrm{n})}=\frac{0.0015}{0.01}=\right.$ $0.15] \rightarrow\left[\frac{0.001636}{0.01}=0.1636\right]$. La productividad neta del capital se eleva en $\left[\hat{y}_{K}=\left|\lambda_{K}-k\right| \therefore\right.$ $\left.\dot{y}_{K}=|0.0015-0.01|=0.0085\right] \rightarrow\left[\dot{y}_{K}=|0.001636-0.012|=0.0104\right]$ y su contribución relativa al ingreso cae en $\left[\frac{\lambda_{K}}{(\mathrm{r}, \mathrm{k})}=\frac{0.0015}{0.01}=0.15\right] \rightarrow\left[\frac{0.001636}{0.012} \cong 0.1364\right]$. En convergencia ocurre divergencia: $\quad \alpha=\mathrm{r} \beta<\mathrm{s}(1-\beta) \quad$ para que $[\alpha=(0.3)(0.45 \overline{45}) \cong 0.1364<(0.3)(1-$ $0.45 \overline{45}) \cong 0.1636]$; lo que es lo mismo: $\left[\frac{\lambda_{K}}{(\dot{r}, \mathrm{k})}=0.1364<\frac{\lambda_{T}}{(\dot{\mathrm{s}}, \mathrm{n})}=0.1636\right]$.

Además, $\pi=\frac{0.1364}{1-0.1364} \cong 0.1579 \cong 16 \%$ del valor de $(Y)$ para el capital y $(1-\pi)=$ $(1-0.1579) \cong 0.842 \cong 84 \%$ para el trabajo. La distribución del crecimiento de $(Y)$ es $\left[\lambda=\lambda_{K}+\lambda_{T} \therefore \lambda \cong 0.0005168+0.002756 \therefore \lambda \cong 0.003273\right]$. La transferencia de valor de producción entre los recursos se computa como $\left[\left(\lambda_{K}-\lambda_{K}\right)=-0.00112 ; \lambda_{T}-\lambda_{T}=0.00112\right]$, y se logra equilibrio entre el crecimiento del producto y su distribución o bien que prevalece la convergencia y divergencia con equidad. Por la ecuación (44), [ú $=0.002=j$ ] y $(v)$ se desplaza proporcional a $(\mathrm{J})$, por encima del instante inicial. Por la función anterior: $0.003273=[(0.002 v) ;(v, 1) 0.01](0.3)(0.45 \overline{45}) \therefore\left[v \cong 1.2=\frac{\hat{r}}{s}=\mathrm{J}\right] . \quad$ La mejora en la viabilidad tecnológica y aumentos proporcionales de la tasa de retorno y del salario respecto de la tasa de inversión en capital e incremento en el trabajo respectivamente, elevan la participación del trabajo en el crecimiento del producto y reducen proporcionalmente la del capital. La divergencia operaría como mecanismo inherente de la convergencia para la consecución de la equidad (Figura 2). 


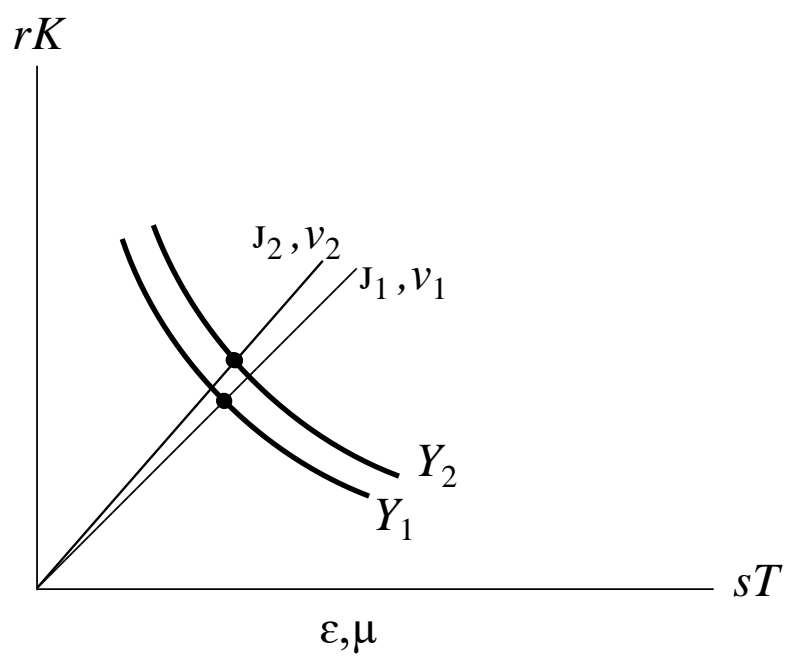

Figura 2. Convergencia y divergencia con equidad. Nivel y distribución de $Y$ dado $(\mu: \mu=1 \rightarrow \mu=$ $\left.\frac{5}{6}\right) ;\left(\beta: \beta=\frac{1}{2} \rightarrow ß=0.45 \overline{45}\right)$ y su efecto en $v, \mathrm{~J}$.

En la Figura 2, la equidad surge porque la elasticidad-de-distribución del crecimiento del valor de producción converge con el cambio en la elasticidad-de-composición de los recursos ceteris paribus: $\left(\pi=\frac{\dot{s}+0.01}{\dot{\mathrm{r}}+0.012}>\mu=0.8 \overline{3}\right) \therefore(\alpha<\beta)$. El valor de $(\mu)$ se indica por la pendiente de las curvas de valor de producción $\left(Y_{1}, Y_{2}\right)$, mientras el valor $\pi$ se mide por la distancia entre ambas curvas en los puntos de corte de los vectores $\left(\mathrm{J}_{1}, v_{1} \rightarrow \mathrm{J}_{2}, v_{2}\right)$. Si en esa economía sucede, en un instante posterior al cambio en la viabilidad tecnológica, un alza en el salario ceteris paribus, aumenta el costo de producción. El efecto de ś es, ceteris paribus, estimular una re-distribución de $(Y)$, alterando el proceso de convergencia y divergencia con equidad.

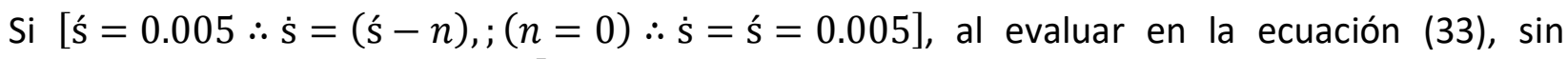
|valor absoluto| del indicador, $\left[\beta=-\frac{(\dot{\mathrm{s}}, n) \mathrm{s}}{(\dot{\mathrm{r}}, k) \mathrm{r} ;(\dot{\mathrm{s}}, n) \mathrm{s}} \therefore \beta=-\frac{\dot{\mathrm{s} s}}{\dot{\mathrm{r}} ; \dot{\mathrm{s} s}} \therefore 0.45 \overline{45}=-\frac{(0.005)(0.3)}{\dot{\mathrm{r}}(0.30),(0.005)(0.3)} \therefore\right.$ $\dot{\mathrm{r}}=-0.006]$ y $[(\dot{\mathrm{r}}=\dot{\mathrm{r}}-\mathrm{k}) ;(k=0) \therefore(\dot{\mathrm{r}}=\dot{\mathrm{r}}=-0.006)]$ y resulta de esa acción $[\dot{\mathrm{s}}=0.005>$ ŕ $=-0.006]$.

Por la función (31) - o (37)-: $[\lambda=f[(-0.006)(0.45 \overline{45})(0.3) ;(0.005)(0.3)(1-0.45 \overline{45})] \therefore \lambda=$ $-0.0008181+0.0008181=0 \therefore \lambda=f(0)=0]$.

Variaciones en (ś, ŕ) no afectan la productividad de los recursos, $\left(\lambda_{K}, \lambda_{T}\right)=0$, pero sí su distribución. El cambio en el retorno no tiene impacto en los costos, pero el ingreso del trabajo es a la vez costo del trabajo. (ś, ŕ) ceteris paribus provocan una disminución de $r$ : $\left[r_{2}=\right.$ $\left.r_{1}(1-0.006)=0.3(1-0.006)=0.2982\right]$ y $\alpha=(0.2982)(0.45 \overline{45}) \cong 0.1355$, o bien: $\alpha_{3}=$ 
$\alpha_{2}-0.0008182 \therefore \alpha_{3}=0.1364-0.0008182 \cong 0.1355 \mathrm{y} \pi \cong 0.1567$. Respecto de la situación previa la distribución del crecimiento del valor de producción es:

$\left[\chi_{K}=\pi \lambda=(0.1567)(0.003273)=0.0005129 ; \lambda_{T}=(1-\pi) \lambda=(0.8433)(0.003273)=\right.$ $0.00276]$.

La transferencia de valor de producción es $\left[\lambda_{K}-\lambda_{K} \cong-0.001123 ; \lambda_{T}-\lambda_{T} \cong 0.001123\right]$. Ocurre divergencia con inequidad; el capital registra una pérdida de retorno equivalente al exceso de ingreso del trabajo.

Por la ecuación (44) v́ $=(-0.006-0.005) \therefore$ v́ $=-0.011$ y se registra un movimiento descendente de $v$ sobre la curva $(Y)$ y su magnitud del valor indicada por el signo negativo $(-)$. La oscilación de $v$ se traza por debajo de la ruta previa al alza en el salario e indica una distorsión en la divergencia, al inducir inequidad (Figura 3).

Por la función (11) $[\lambda=f[(0.1364)(-0.006),(1-0.1364)(0.006)(0.1579)] \therefore \lambda=$ $-0.0008181+0.0008181=0]$.

La función (11) oculta la diferencia efectiva en la magnitud de (ś, ŕ), no así la dirección inversa; son variables mutuamente dependientes e inclusivas, y reflejan sus oscilaciones sobre la curva de $(Y)$, congruente con la función (31). La divergencia con inequidad emerge cuando la elasticidadde-distribución crecimiento del valor de producción no converge con el cambio en la elasticidadde-composición de los recursos ceteris paribus.

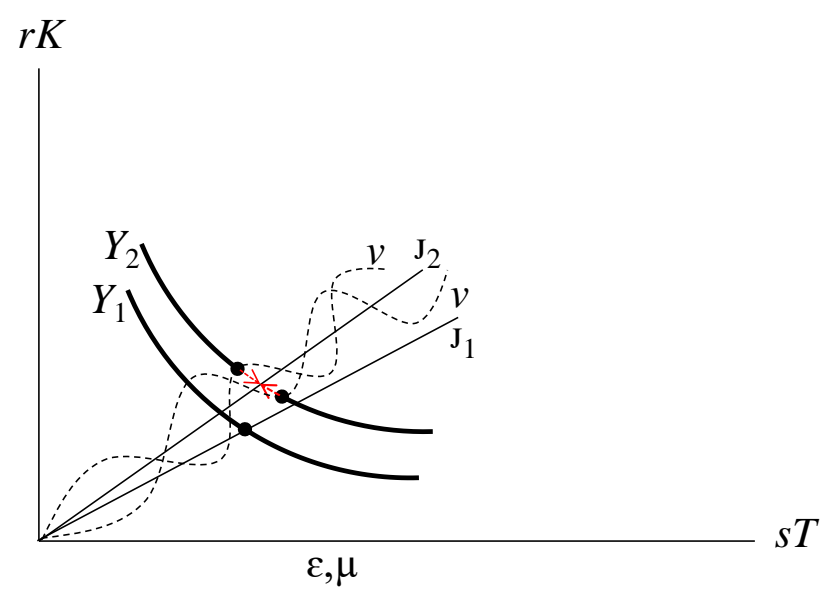

Figura 3. Convergencia y divergencia con inequidad. Oscilación de $v$ en torno a $\mathrm{J}$ dado $\left(\mu: \mu=1 \rightarrow \mu=\frac{5}{6}\right) ;\left(ß: ß=\frac{1}{2} \rightarrow ß=0.45 \overline{45}\right) ;(\dot{\mathrm{r}}=-0.006, \dot{s}=0.005)$.

52 